\title{
Extremely low thermal conductivity from bismuth selenohalides with 1D soft crystal structure
}

\author{
Dongyang Wang ${ }^{1 \dagger}$, Zhiwei Huang ${ }^{1 \dagger}$, Yang Zhang ${ }^{3,4}$, Lijie Hao ${ }^{5}$, Guangtao Wang ${ }^{6}$, Sihao Deng ${ }^{7}$, \\ Hongliang Wang ${ }^{5}$, Jie Chen ${ }^{9}$, Lunhua $\mathrm{He}^{7,8,9}$, Bao Xiao ${ }^{10}$, Yadong Xu ${ }^{10}$, Stephen J. Pennycook ${ }^{4}$, \\ Haijun $\mathrm{Wu}^{2,4^{*}}$ and Li-Dong Zhao ${ }^{1^{*}}$
}

\begin{abstract}
Materials with intrinsically low thermal conductivity are of fundamental interests. Here we report a new sort of simple one-dimensional (1D) crystal structured bismuth selenohalides (BiSeX, $\mathrm{X}=\mathrm{Br}, \mathrm{I}$ ) with extremely low thermal conductivity of $\sim 0.27 \mathrm{~W} \mathrm{~m}^{-1} \mathrm{~K}^{-1}$ at $573 \mathrm{~K}$. The mechanism of the extremely low thermal conductivity in 1D BiSeX is elucidated systematically using the first-principles calculations, neutron powder-diffraction measurements and temperature tunable aberration-corrected scanning transmission electron microscopy (STEM). Results reveal that the 1D structure of BiSeX possesses unique soft bonding character, low phonon velocity, strong anharmonicity of both acoustic and optical phonon modes, and large off-center displacement of $\mathrm{Bi}$ and halogen atoms. Cooperatively, all these features contribute to the minimal phonon transport. These findings provide a novel selection rule to search low thermal conductivity materials with potential applications in thermoelectrics and thermal barrier coatings.
\end{abstract}

Keywords: low thermal conductivity, 1D bulk material, bismuth selenohalides, first-principles calculations

\section{INTRODUCTION}

Extreme heat transport properties are important for applications in thermal management, including high ther- mal conductivity for heat transmission and dissipation [1], and low thermal conductivity for thermal barrier coatings [2-4] and thermoelectrics [5-9]. The thermal conductivity of a material is mainly determined by the atomic vibrations of the crystal lattice, especially for insulator. The commonly accepted criteria for high thermal conductivity are simple crystal structure, low molecular weight, strong chemical bonding and low anharmonicity [1,10-13]. In contrast, materials with intrinsically low thermal conductivity can be rationally designed with the opposite attributes of complex crystal structure [14,15], high molecular weight, large unit cell and strong anharmonicity [16-21]. In this paper, we report one type of materials, bismuth selenohalides, which possess extremely low thermal conductivity. It is remarkable that a low thermal conductivity is found in such a simple compound as BiSeX ( $\mathrm{X}=\mathrm{I}$ and $\mathrm{Br}$ ), which does not obey the accepted selection rules for low thermal conductivity. To elucidate the origins of low thermal conductivity, we make comparisons with several analogues of bismuth selenohalides, which exhibit cubic- (three-dimensional (3D)), layer(2D) and chain-like (1D) crystal structures, respectively. We find that the thermal conductivity shows a decreasing trend from $3 \mathrm{D}$ to $2 \mathrm{D}$, and to $1 \mathrm{D}$, which is due to the chemical bonding along the three crystallographic axial

\footnotetext{
${ }^{1}$ School of Materials Science and Engineering, Beihang University, Beijing 100191, China

2 State Key Laboratory for Mechanical Behavior of Materials, Xi'an Jiaotong University, Xi'an 710049, China

${ }^{3}$ Instrumental Analysis Center of Xi'an Jiaotong University, Xi'an Jiaotong University, Xi'an 710049, China

${ }^{4}$ Department of Materials Science and Engineering, National University of Singapore, Singapore 117575, Singapore

${ }^{5}$ Department of Nuclear Physics, Neutron Scattering Laboratory, China Institute of Atomic Energy, Beijing 102413, China

${ }^{6}$ School of Physics, Henan Normal University, Xinxiang 453007, China

${ }^{7}$ Spallation Neutron Source Science Center, Dongguan 523803, China

${ }^{8}$ Beijing National Laboratory for Condensed Matter Physics, Institute of Physics, Chinese Academy of Sciences, Beijing 100190, China

${ }^{9}$ Songshan Lake Materials Laboratory, Dongguan 523808, China

${ }^{10}$ State Key Laboratory of Solidification Processing, Northwestern Polytechnical University, Xi'an 710072, China

$\uparrow$ These authors contributed equally to this work.

* Corresponding authors (emails: wu.haijun@u.nus.edu (Wu H); zhaolidong@buaa.edu.cn (Zhao LD))
} 
directions becoming progressively weaker and weaker. Based on these guidelines, we further find these bismuth selenohalides have not only a $1 \mathrm{D}$ crystal structure, but also weaker chemical bonding along the 1D direction due to large off-center displacements of Bi and halogen atoms. This soft $1 \mathrm{D}$ crystal structure means that the chemical bonding along all three crystallographic directions is weak, therefore resulting in a quasi-0D crystal structure [22]. Therefore, bismuth selenohalides exhibit extremely low thermal conductivity since phonons cannot find strong transport pathways in such loose crystal structures, which is in contrast to diamond with high thermal conductivity due to strong chemical bonding [1]. Our findings may offer a promising route to find potential materials with low thermal conductivity in the field of thermal barrier coatings [2-4], thermoelectric materials [5-8,23], and other applications.

Here, bismuth selenohalides were successfully synthesized by ball milling and spark plasma sintering (SPS) methods (Fig. S1) and found an extremely low thermal conductivity of $\sim 0.27 \mathrm{~W} \mathrm{~m}^{-1} \mathrm{~K}^{-1}$ at $573 \mathrm{~K}$ (Fig. 1 and Fig. S2). Total thermal conductivity $\left(\kappa_{\text {tot }}\right)$ is a sum of lattice thermal conductivity $\left(\kappa_{\text {lat }}\right)$ and electronic thermal conductivity $\left(\kappa_{\text {ele }}\right) \cdot \kappa_{\text {tot }}$ is similar to $\kappa_{\text {lat }}$, if $\kappa_{\text {ele }}$ can be ignored due to poor electrical conductivity. Experimentally, the origins of low thermal conductivity have been investigated by comparing the crystal structure and temperature-dependent atomic vibrations in $2 \mathrm{D} \mathrm{Bi}_{2} \mathrm{Se}_{3}$ and $1 \mathrm{D} \mathrm{Sb} \mathrm{Se}_{3}$ through neutron powder-diffraction, combined with the first-principles calculations. The unique 1D structure with weak bonding, low phonon velocity, strong anharmonicity of both acoustic and optical phonon modes, and the large atomic displacement of $\mathrm{Bi}$ and halogens collectively lead to the extremely low thermal conductivity in the $1 \mathrm{D}$ chain-like bismuth selenohalides with relatively simple unit cell.

\section{EXPERIMENTAL SECTION}

\section{Materials synthesis}

High purity raw materials were selected as starting materials for the synthesis of the samples. The bulk samples of polycrystalline were prepared by ball milling and SPS methods. The temperature gradient method was used to grow $\mathrm{Sb}_{2} \mathrm{Se}_{3}$ and $\mathrm{Bi}_{2} \mathrm{Se}_{3}$ crystals. The BiSeI crystals were grown using the vapor phase transport method. The optical images of the crystals can be found in Fig. S3. The detailed information can be found in the Supplementary information.

\section{Material characterization}

The powder X-ray diffraction (PXRD) patterns were obtained by using a D/max 2200PC diffractometer with $\mathrm{Cu}$ Ka1 $(\lambda=1.5405 \AA)$ radiation. The aberration-corrected scanning transmission electron microscopy (STEM) and in-situ STEM heating experiment were performed using a Gatan double tilting heating holder on the JEOL ARM200F. Neutron powder-diffraction measurements were performed on the general-purpose powder diffractometer (GPPD) at China Spallation Neutron Source (CSNS) and then the Rietveld refinement of the crystal structures was performed. The ion impedance measurements were conducted using an electrochemical workstation (CHI600E, Shanghai Chenhua). The thermal diffusivity $(D)$ was measured by a laser flash analysis system (LFA457, Netzsch) under $\mathrm{N}_{2}$ atmosphere at $300-573 \mathrm{~K}$ and analyzed with Cowan model. The sample density $(\rho)$ was determined by using the dimensions and mass of the sample and then reconfirmed by using a gas pycnometer instrument (Micromeritics AccuPyc1340).
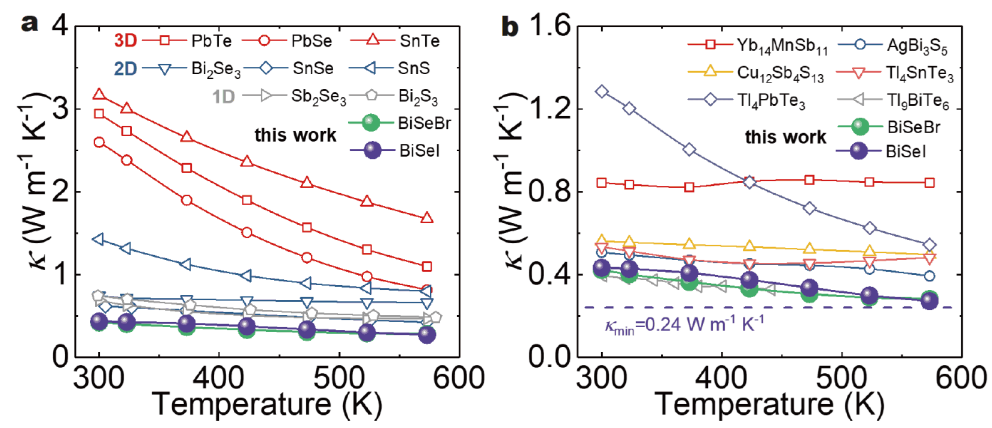

Figure 1 Experimental thermal conductivities as a function of temperature. (a) The thermal conductivities of IV-VI compounds show a decreasing trend as the crystal structure dimension decreases from 3D, 2D to 1D, and the thermal conductivity of BiSeBr and BiSeI are further reduced due to the 1D soft crystal structures from halogen atoms. (b) Comparison of the thermal conductivity of the present bismuth selenohalides with various compounds exhibiting intrinsically low thermal conductivity. The dashed line refers to the theoretical minimal thermal conductivity of BiSeI. 
The electrical conductivity $(\sigma)$ and Seebeck coefficients $(S)$ were measured by using the four-probe method with Cryoall CTA and Ulvac Riko ZEM-3 instrument under a low-pressure helium atmosphere. The optical band gap measurements were performed by using a Shimadzu Model UV-3600 Plus equipped with double beam, double-monochromator spectrophotometer (ultravioletvisible spectroscopy). The longitudinal and transverse speed of sound was measured by using an ultrasonic instrument (Ultrasonic Pulser/Receiver Model 5058 PR, Olympus, USA). The phonon spectrum, electron localization functions and elastic constant were obtained by using the first-principles calculations.

\section{RESULTS AND DISCUSSION}

The temperature-dependent thermal conductivities of $\operatorname{BiSeX}(\mathrm{X}=\mathrm{I}$ and $\mathrm{Br})$ and its analogues are compared in Fig. 1a. 3D cubic-type structures of $\mathrm{PbTe}$ $\left(\sim 2.94 \mathrm{~W} \mathrm{~m}^{-1} \mathrm{~K}^{-1}\right)$ [24], $\mathrm{PbSe}\left(\sim 2.60 \mathrm{~W} \mathrm{~m}^{-1} \mathrm{~K}^{-1}\right)$ [24] and SnTe $\left(\sim 3.16 \mathrm{~W} \mathrm{~m}^{-1} \mathrm{~K}^{-1}\right)$ [24] exhibit relatively high thermal conductivities at $300 \mathrm{~K}$. With decreasing crystal structure dimensionality, the thermal conductivity is clearly decreased due to the enhanced phonon scattering between van der Waals layers, such as $2 \mathrm{D}^{-1} \mathrm{Bi}_{2} \mathrm{Se}_{3}$ $\left(\sim 0.74 \mathrm{~W} \mathrm{~m}^{-1} \mathrm{~K}^{-1}\right), 2 \mathrm{D} \mathrm{SnSe}\left(\sim 0.46 \mathrm{~W} \mathrm{~m}^{-1} \mathrm{~K}^{-1}\right)$ [5] and 2D SnS $\left(\sim 1.42 \mathrm{~W} \mathrm{~m}^{-1} \mathrm{~K}^{-1}\right)$ [25] at $300 \mathrm{~K}$. For the infinite chain containing $1 \mathrm{D} \mathrm{Sb}_{2} \mathrm{Se}_{3}$ and $1 \mathrm{D} \mathrm{Bi}_{2} \mathrm{~S}_{3}$ [26], the interaction between the chains further weakens and the thermal conductivity further declines. With the aid of halogens, weak ionic bonding in $1 \mathrm{D}$ BiSeX leads to the extremely low thermal conductivity. Compared with other reported intrinsically low thermal conductivity materials [14,27-30], bismuth selenohalides exhibit an even lower thermal conductivity without a complex crystal structure, high molecular weight, or large unit cell, and contain no toxic elements, as shown in Fig. 1b. The thermal conductivity of $1 \mathrm{D}$ BiSeI of $0.27 \mathrm{~W} \mathrm{~m}^{-1} \mathrm{~K}^{-1}$ at $573 \mathrm{~K}$ approaches the theoretical minimal thermal conductivity $\left(\kappa_{\min }\right)$.

In order to reveal the origins of this extremely low thermal conductivity, the structure differences among $2 \mathrm{D}$ $\mathrm{Bi}_{2} \mathrm{Se}_{3}, 1 \mathrm{D} \mathrm{Sb}_{2} \mathrm{Se}_{3}$ and $1 \mathrm{D}$ BiSeI were investigated. Fig. 2a$c$ show the schematic structure of each compound, where the arrows along the chain refer to the migration of halogens (Fig. 2c). Clearly, in the $\mathrm{Bi}_{2} \mathrm{Se}_{3}$ crystal in 2D rhombohedral structure, five independent atoms form a layer with Bi-Se bonding (Fig. 2d), which are then stacked along the $b$ direction with van der Waals interaction (Fig. 2g). $\mathrm{Sb}_{2} \mathrm{Se}_{3}$ belongs to the 1D orthorhombic structure, with covalent $\mathrm{Sb}-\mathrm{Se}$ bonds forming chains along the $c$ direction (Fig. 2e), which are stronger than the inter chain interaction along $a$ and $b$ directions (Fig. 2h). Similarly, covalent $\mathrm{Bi}-\mathrm{Se}$ and ionic Bi-I bonds form infinite chains (Fig. 2f), which comprise the 1D BiSeI crystal with transformation and rotation (Fig. 2i).

To directly demonstrate the structure difference of 1D bismuth selenohalides compared with $2 \mathrm{D} \mathrm{Bi}_{2} \mathrm{Se}_{3}$ and $1 \mathrm{D}$ $\mathrm{Sb}_{2} \mathrm{Se}_{3}$, the aberration-corrected STEM was employed to reveal their crystal structures and quantitatively analyze the bond lengths, i.e., bond strength. The 1D morphology of bismuth selenohalides can be reflected in Fig. 3. It is possible to find nanowires in bulk BiSeI, while none exist in $2 \mathrm{D} \mathrm{Bi}_{2} \mathrm{Se}_{3}$ and $1 \mathrm{D} \mathrm{Sb}_{2} \mathrm{Se}_{3}$. Actually, the $1 \mathrm{D}$ nanowire morphology could reflect the soft $1 \mathrm{D}$ characteristic of bismuth selenohalides at the unit cell level. BiSeBr and BiSeI display the same crystal structure, as shown in Fig. 3a and its inset, but it is difficult to differentiate $\mathrm{Se}(Z$ $=34)$ and $\operatorname{Br}(Z=35)$ atoms, thus BiSeI (three elements locating on different periods) is used as the example to decode the crystal structure of bismuth selenohalides. Fig. $4 \mathrm{a}-\mathrm{c}$ present atomically-resolved STEM high-angle annular dark-field (HAADF) images of BiSeI along $c, b$ and $a$ directions, i.e., [001], [010] and [100] zone axes, respectively. The experimentally acquired structural images of BiSeI are well consistent with the proposed structural model. For comparison, Fig. $4 \mathrm{~d}-\mathrm{f}$ show the structural images of $\mathrm{Sb}_{2} \mathrm{Se}_{3}$ and $\mathrm{Bi}_{2} \mathrm{Se}_{3}$ along the respective zone axes. To quantitatively compare the corresponding bond lengths of the three materials, firstly, atoms with different bonding surroundings were grouped, as marked in Fig. $4 a_{2}-f_{2}$, secondly, the atom column positions were accurately located through 2D Gaussian fitting $[31,32]$, thirdly, bond lengths of many equivalent bonds in one large image were calculated, and finally, the average bond lengths and the respective deviations $(\sim 5 \%)$ were obtained. Table S1 summarizes the bond lengths for BiSeI, $\mathrm{Sb}_{2} \mathrm{Se}_{3}$, and $\mathrm{Bi}_{2} \mathrm{Se}_{3}$ along [100]/[010]/[001]. We compare the bond lengths of $\mathrm{Bi}-\mathrm{Se} / \mathrm{I}$ for BiSeI with $\mathrm{Bi}-\mathrm{Se}$ for $\mathrm{Bi}_{2} \mathrm{Se}_{3}$ and $\mathrm{Sb}-\mathrm{Se}$ for $\mathrm{Sb}_{2} \mathrm{Se}_{3}$ along the same zone axis. It should be noted that the bond lengths obtained from the STEM images are the real bond lengths projected into the observation plane. Table S2 compares the experimentally obtained bond lengths of the three materials along three base axes, with those obtained by density functional theory (DFT) calculation. Good agreement is obtained. For bonds of similar type, the bond length directly reflects the bond strength. Therefore, the bond strengths of the three materials along three base axes are revealed as: along the $a$ axis, $\mathrm{BiSeI} \approx \mathrm{Sb}_{2} \mathrm{Se}_{3}<\mathrm{Bi}_{2} \mathrm{Se}_{3}$; along the $b$ axis, BiSeI $\approx \mathrm{Sb}_{2} \mathrm{Se}_{3} \approx \mathrm{Bi}_{2} \mathrm{Se}_{3}$; along the $c$ axis, BiSeI $<\mathrm{Sb}_{2} \mathrm{Se}_{3} \approx$ 
a

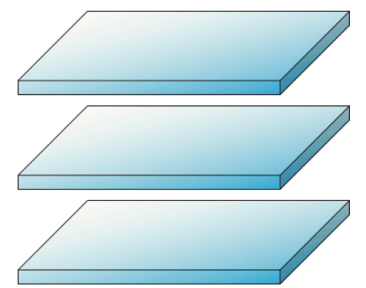

d

$\mathrm{Bi} \mathrm{Se}$

o- -orecer

- $-000-000$

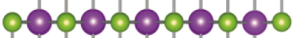

$0-000-000$

0000000000

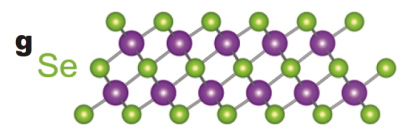

bBiggogogo

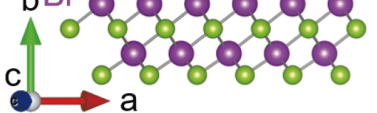

j

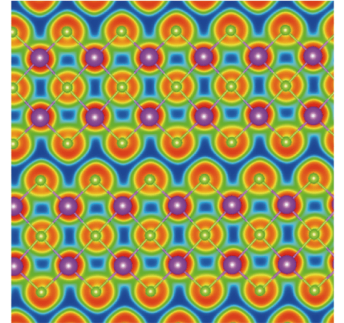

b

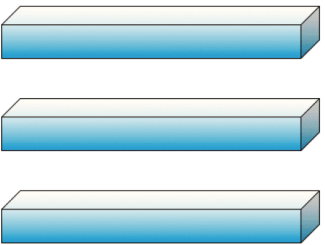

$\mathrm{Sb} \mathrm{Se}$

e 000000000000 000000000000

00000000000 000000000000

000000000000

000000000000

h

की 8000

Se \&ra
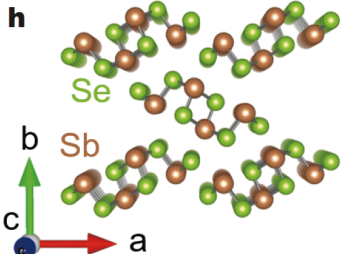

$\mathbf{k}$

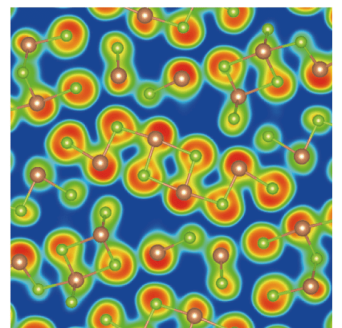

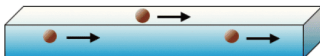
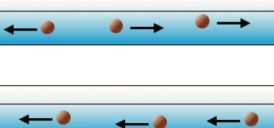

$\mathrm{Bi} \mathrm{Se}$

f 0000000090 100000000000 0000000000 0000000000 0000000000 0.0000000000

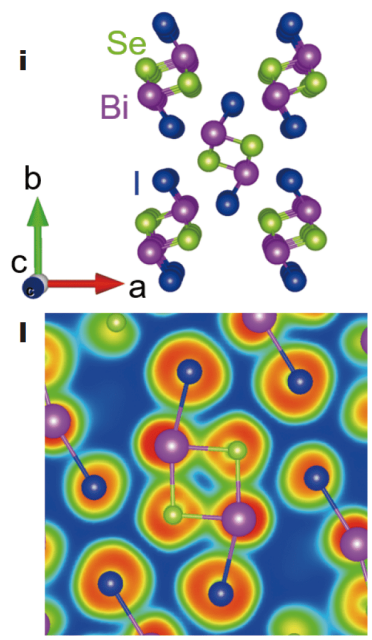

Figure 2 Schematic crystal structures and electronic localization functions (ELFs) of 2D, 1D, and soft $1 \mathrm{D} \mathrm{Bi}_{2} \mathrm{Se}_{3}, \mathrm{Sb}_{2} \mathrm{Se}_{3}$ and $\mathrm{BiSeI}$, respectively. Schematic diagrams and the corresponding crystal structures of (a, d) $2 \mathrm{D}$ slabs in $\mathrm{Bi}_{2} \mathrm{Se}_{3}$, (b, e) $1 \mathrm{D}$ chain in $\mathrm{Sb}_{2} \mathrm{Se}_{3}$ and (c, f) 1D chain with migration of halogens in BiSeI. The crystal structures of $\mathrm{Bi}_{2} \mathrm{Se}_{3}, \mathrm{Sb}_{2} \mathrm{Se}_{3}$ and BiSeI viewed along the $c$ direction are given in (g-i), respectively. (j-l) The projected ELF along the chain. The isosurface level of ELF is 0.9 .

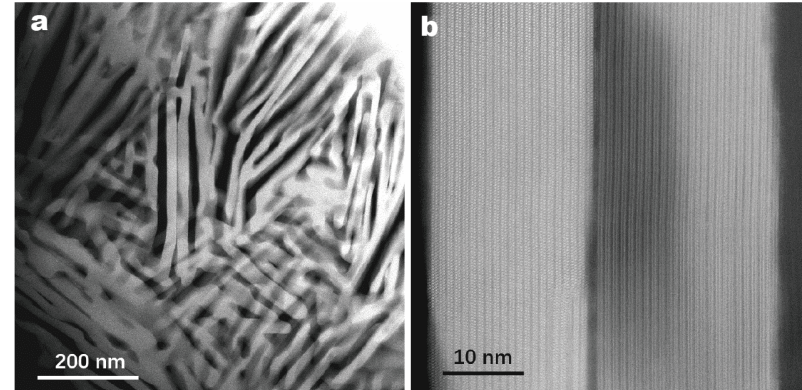

Figure 3 The morphology of BiSeI. (a) Wires shape and (b) parallel chains in soft $1 \mathrm{D}$ BiSeI.

$\mathrm{Bi}_{2} \mathrm{Se}_{3}$. Therefore, the soft $1 \mathrm{D}$ BiSeI displays the weakest bonding, especially along the $1 \mathrm{D}$ chain, compared with the $2 \mathrm{D} \mathrm{Bi}_{2} \mathrm{Se}_{3}$ and $1 \mathrm{D} \mathrm{Sb} \mathrm{Se}_{3}$. The in-situ heating on the aberration-corrected STEM was further employed to test the temperature-dependent change of bond lengths for BiSeI and $\mathrm{Bi}_{2} \mathrm{Se}_{3}$, as shown in Fig. $5 \mathrm{a}_{1}-\mathrm{a}_{3}$ and $\mathrm{b}_{1}-\mathrm{b}_{3}$, respectively. Fig. $5 \mathrm{c}$ displays the temperature-dependent change of $\mathrm{Bi}-\mathrm{Bi}$ and $\mathrm{Bi}-\mathrm{Se}$ bond lengths relative to the values at room temperature for both $\mathrm{BiSeI}$ and $\mathrm{Bi}_{2} \mathrm{Se}_{3}$. It is apparent that BiSeI shows much larger relative off-center displacement than $\mathrm{Bi}_{2} \mathrm{Se}_{3}$, especially for $\mathrm{Bi}-\mathrm{Se}$ bonds, $\sim 38 \%$ increase in BiSeI compared with $\sim 12 \%$ for $\mathrm{Bi}_{2} \mathrm{Se}_{3}$.

To elucidate the bonding character of these compounds, the DFT was also used to calculate the electronic localization functions (ELFs) [33] based on the optimized cell parameters (Table S3). The projected ELFs are shown in Fig. 2j, k. Electrons overlap only in the inter-layer and inter-chain regions, indicating the weaker van der Waals interaction between the neighboring layers and chains. The interstitial electrons between $\mathrm{Bi} / \mathrm{Sb}$ and Se localize close to Se atoms, since the Pauling electronegativity of 


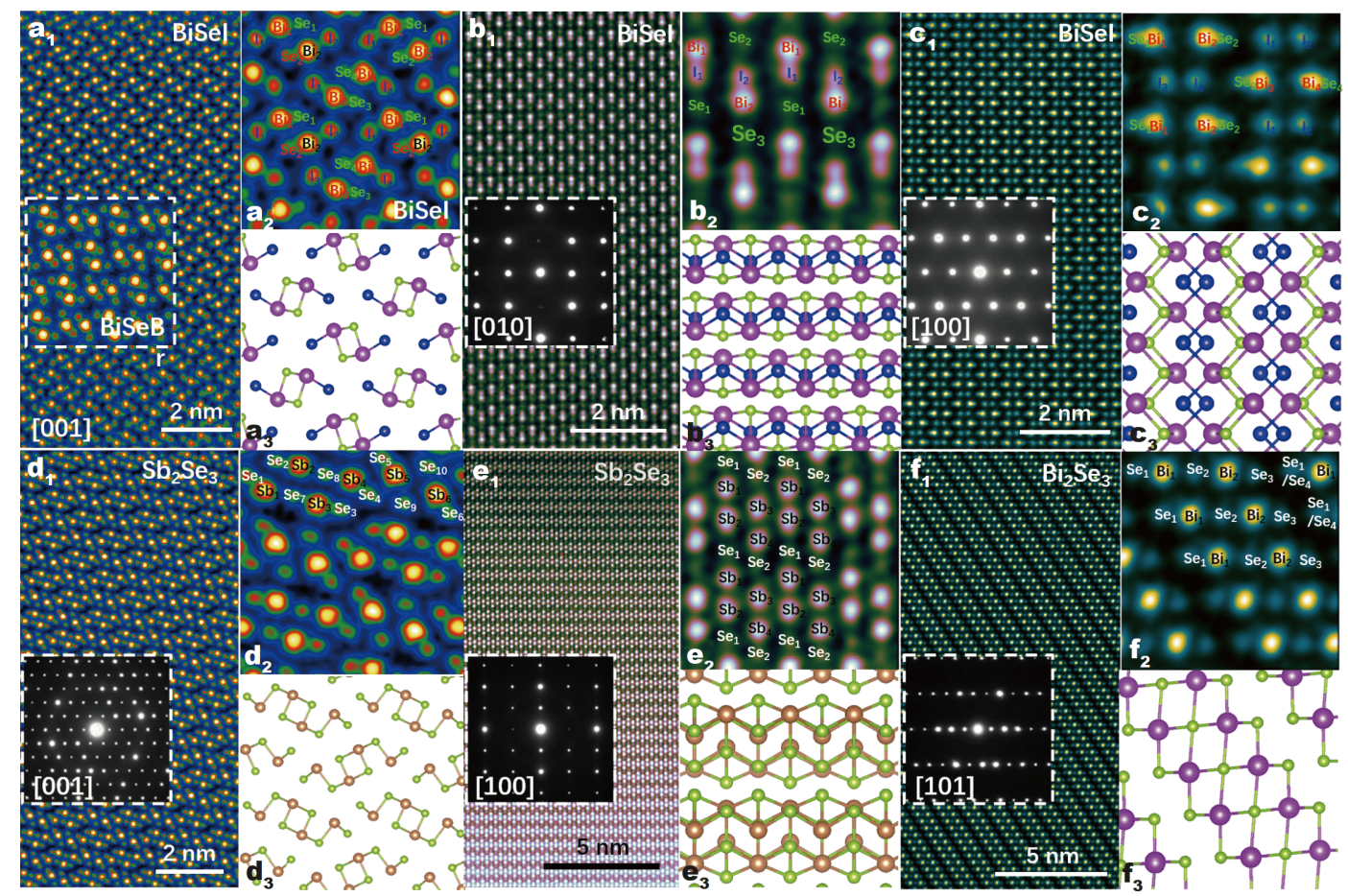

Figure 4 Crystal structures and bond lengths of $2 \mathrm{D} \mathrm{Bi}_{2} \mathrm{Se}_{3}, 1 \mathrm{D} \mathrm{S} \mathrm{Sb}_{2}$, and soft $1 \mathrm{D}$ BiSeI/BiSeBr. $\left(\mathrm{a}_{1}, \mathrm{~b}_{1}, \mathrm{c}_{1}\right)$ Atomically-resolved STEM HAADF images of BiSeI along [001], [010], [100] zone axes, respectively. The inset in $\left(a_{1}\right)$ is an enlarged STEM HAADF image of BiSeBr along the [001] zone axis. The insets in $\left(b_{1}, c_{1}\right)$ are electron diffraction patterns. $\left(a_{2}, b_{2}, c_{2}\right)$ Enlarged STEM HAADF images from $\left(a_{1}, b_{1}, c_{1}\right)$, respectively, where different atoms are marked. $\left(a_{3}, b_{3}, c_{3}\right)$ Structural models along [001], [010], [100] zone axes, which are consistent with $\left(a_{2}, b_{2}, c_{2}\right) .\left(d_{1}, e_{1}\right)$ Atomically-resolved STEM HAADF images of $\mathrm{Sb}_{2} \mathrm{Se}_{3}$ along [001] and [100] zone axes, respectively, with electron diffraction patterns insets. $\left(d_{2}, e_{2}\right)$ Enlarged STEM HAADF images from $\left(d_{1}\right)$ and $\left(e_{1}\right)$, respectively, where different atoms are marked. $\left(d_{3}, e_{3}\right)$ Structural models along [001] and [100] zone axes, which are consistent with $\left(\mathrm{d}_{2}\right)$ and $\left(\mathrm{e}_{2}\right)$. ( $\left.\mathrm{f}_{1}\right)$ Atomically-resolved STEM HAADF image of $\mathrm{Bi}_{2} \mathrm{Se}_{3}$ along the [101] zone axis, with electron diffraction pattern inset. $\left(f_{2}\right)$ Enlarged STEM HAADF image from $\left(f_{1}\right)$, where different atoms are marked. $\left(f_{3}\right)$ Structural model along the [101] zone axeis, which is consistent with $\left(\mathrm{f}_{2}\right)$.

Se (2.4) is larger than that in $\mathrm{Bi}(2.02)$ and $\mathrm{Sb}$ (2.05). The covalent bond of $\mathrm{Bi}_{2} \mathrm{Se}_{3}$ along the $a$ direction is stronger than that of van der Waals interaction in $\mathrm{Sb}_{2} \mathrm{Se}_{3}$ along the $a$ direction, while the overall interaction strength weakens from $\mathrm{Bi}_{2} \mathrm{Se}_{3}(2 \mathrm{D})$ to $\mathrm{Sb}_{2} \mathrm{Se}_{3}(1 \mathrm{D})$. The bond lengths of $\mathrm{Bi}-$ Se $(2.849 \AA)$ and $\mathrm{Bi}-\mathrm{I}(3.227 \AA)$ are relatively larger than that of $\mathrm{Sb}-\mathrm{Se}(2.712$ and $2.787 \AA)$ in $\mathrm{Sb}_{2} \mathrm{Se}_{3}$, suggesting the weak bonding of bismuth selenohalides along the $1 \mathrm{D}$ chain. Therefore, both the van der Waals interaction and the weak bonding along the chain contribute to the extremely low thermal conductivity of bismuth selenohalides.

In kinetic theory, the thermal conductivity $\kappa_{\text {lat }}$ of bulk materials can be expressed as $\kappa_{\text {lat }}=1 / 3 C_{\mathrm{v}} \cdot v_{\mathrm{g}} \cdot l=1 / 3 C_{\mathrm{v}} \cdot v_{\mathrm{g}}^{2} \cdot \tau$, where $C_{\mathrm{v}}, v_{\mathrm{g}}, l$ and $\tau$ are the specific heat, phonon group velocity, mean-free path and phonon lifetime [34,35]. Thus, low $\kappa_{\text {lat }}$ requires minimal phonon velocity and phonon lifetime. The acoustic and optical branches are coupled around the Brillouin zone boundaries (Fig. S4) and result in strong acoustic-optical interactions and highly nonlinear dispersion curves, which lead to a decrease of group velocity (Fig. S5). The averaged maximum acoustic phonon frequency (Fig. 6a) and averaged group velocity (Fig. 6b) of acoustic branches along three crystallographic axes (Table S4) were extracted. The averaged maximum acoustic phonon frequencies of the bismuth selenohalides along the $a$ and $b$ directions are smaller than that in the $c$ direction, indicating the softer phonon mode due to the weak interaction between the chains (Fig. 6a). Moreover, the averaged maximum acoustic phonon frequency and phonon group velocity and the elastic constants (Tables S5 and S6) decline from 2D Bi $\mathrm{Se}_{3}$, to $1 \mathrm{D} \mathrm{Sb} \mathrm{Se}_{3}$ and $1 \mathrm{D}$ bismuth selenohalides in both $a$ (perpendicular to the chain) and $c$ (parallel to the chain) directions, again indicating the weak bonding along the chain. The experimental phonon velocity and the deduced Young's and shear moduli and Debye temperature of bismuth selenohalides are smaller than that in $2 \mathrm{D} \mathrm{Bi}$ $\mathrm{Se}_{3}$ and $1 \mathrm{D} \mathrm{Sb}_{2} \mathrm{Se}_{3}$ (Table S6), resulting in the low thermal conductivity in bismuth selenohalides according to the 

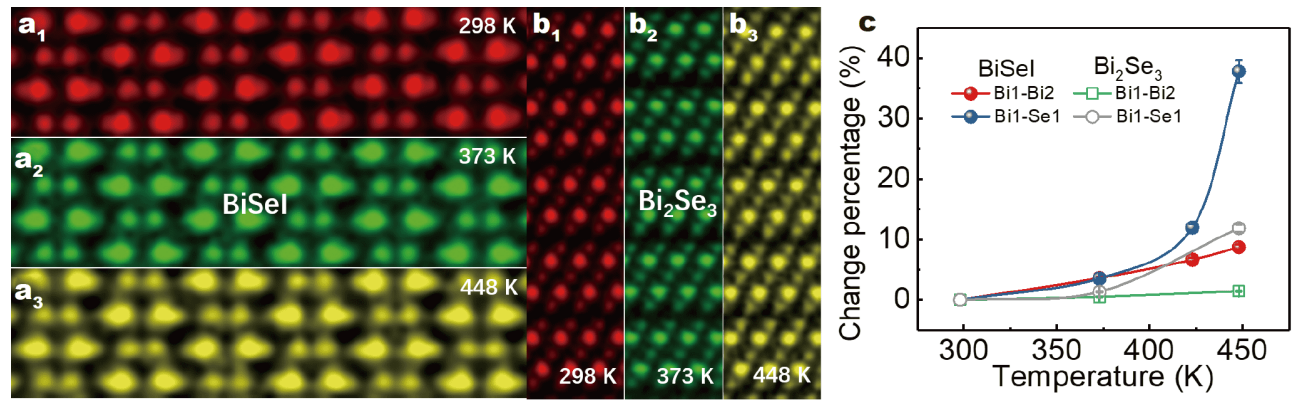

Figure 5 Temperature-dependent atom displacement. STEM HAADF images of $\left(\mathrm{a}_{1}-\mathrm{a}_{3}\right)$ BiSeI and $\left(\mathrm{b}_{1}-\mathrm{b}_{3}\right) \mathrm{Bi}_{2} \mathrm{Se}_{3}$ acquired during in-situ heating at 298,373 and $448 \mathrm{~K}$, respectively. (c) Temperature-dependent $\mathrm{Bi}-\mathrm{Bi}$ and $\mathrm{Bi}-\mathrm{Se}$ bond length changes in BiSeI and $\mathrm{Bi}_{2} \mathrm{Se}_{3}$ with respect to those at $298 \mathrm{~K}$.
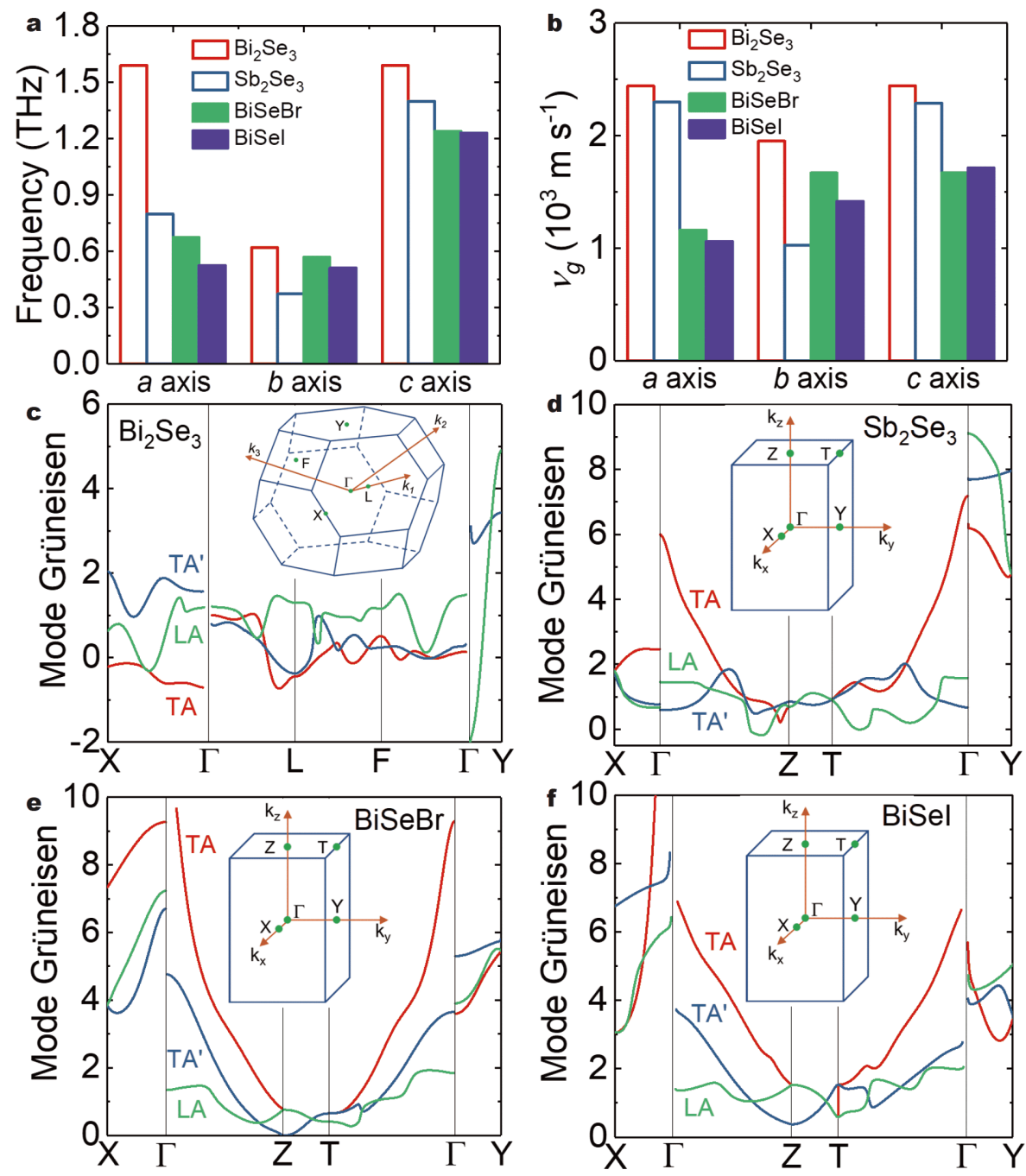

Figure 6 Comparison of the averaged maximum acoustic phonon frequency and phonon group velocity along three crystallographic axes, and Grüneisen dispersions. Averaged (a) maximum acoustic phonon frequency and (b) phonon velocity of transverse (TA, TA') and longitudinal (LA) acoustic modes along three crystalline axes. Calculated Grüneisen dispersions of (c) $2 \mathrm{D} \mathrm{Bi}_{2} \mathrm{Se}_{3}$, (d) $1 \mathrm{D} \mathrm{Sb}_{2} \mathrm{Se}_{3}$, (e) $1 \mathrm{D} \mathrm{BiSeBr}$, and (f) $1 \mathrm{D}$ BiSeI along high symmetry lines. Insets show the corresponding first Brillouin zone and high symmetry points, elucidating that the thermal transport properties of the materials with $1 \mathrm{D}$ soft structure are favorable. 
Slack model [36].

The existence of lone-pair electrons usually causes strong anharmonicity, which is an indicator of low thermal conductivity $[17,37-40]$. The anharmonicity of lattice vibrations can be evaluated by the Grüneisen parameters [41,42], which reflect the relationship between the phonon frequency and cell volume change. Therefore, the Grüneisen parameters were calculated to reveal the origin of the intrinsically extremely low thermal conductivity. The Grüneisen parameters of $2 \mathrm{D} \mathrm{Bi}_{2} \mathrm{Se}_{3}$ along the $\Gamma$ - $Y$ direction are larger than that along the $\Gamma-X$ and $\Gamma$ $L$ directions, indicating the weaker inter-layer interaction than intra-layer (Fig. 6c). The Grüneisen parameters of $1 \mathrm{D} \mathrm{Sb}_{2} \mathrm{Se}_{3}$ are larger than those of $2 \mathrm{D} \mathrm{Bi}_{2} \mathrm{Se}_{3}$ and smaller than those of $1 \mathrm{D}$ bismuth selenohalides, suggesting the weak interaction and strong anharmonicity in the 1D bismuth selenohalides (Table S4). The averaged Grüneisen parameters along the $a, b$ and $c$ directions are 1.51, 7.04 and 1.54 for $1 \mathrm{D} \mathrm{Sb}_{2} \mathrm{Se}_{3}, 6.25,4.82$ and 1.99 for $1 \mathrm{D}$ $\mathrm{BiSeBr}$, and 5.31, 4.05 and 2.36 for $1 \mathrm{D}$ BiSeI, respectively (Fig. 6d-f and Table S4). In contrast, the Grüneisen parameters are 4.41 for $2 \mathrm{D} \mathrm{SnSe} \mathrm{[5]} \mathrm{and} 1.65$ for 3D PbTe [24], corresponding to the measured thermal con- ductivities of 0.46 and $2.94 \mathrm{~W} \mathrm{~m}^{-1} \mathrm{~K}^{-1}$ at $300 \mathrm{~K}$, respectively. The anomalously large Grüneisen parameters strongly reduce the lifetime due to the increased Umklapp processes, thus decreasing the thermal conductivity.

To get further insight into the phonon vibrations in these compounds, the projected density of phonon states (DPS) was calculated and it was found that the low frequency vibration $(<2.0 \mathrm{THz})$ primarily resulted from the vibration of heavier Bi or Sb atoms (Fig. S6). The peak of the projected DPS indicates the potential local vibration of the corresponding atom. The irreducible representation of vibration modes was analyzed and the frozen phonon potential of the first peak was calculated (Fig. 7). The vibration patterns were inserted according to the correspondence mode. The potential of $2 \mathrm{D} \mathrm{Bi}_{2} \mathrm{Se}_{3}\left(\mathrm{E}_{\mathrm{g}}\right.$ mode) and $\mathrm{Sb}_{2} \mathrm{Se}_{3}\left(\mathrm{~A}_{\mathrm{g}}\right.$ mode) can be well fitted by a quadratic curve, indicating the harmonic vibration behavior. For bismuth selenohalides, the potential of the $A_{g}$ mode is asymmetric and strongly deviates from a quadratic dependence, but can be fitted by including both cubic and quartic terms, manifesting the anharmonic bonding and anisotropic displacement within the chain. The calculated mean-squared displacement (MSD) in-
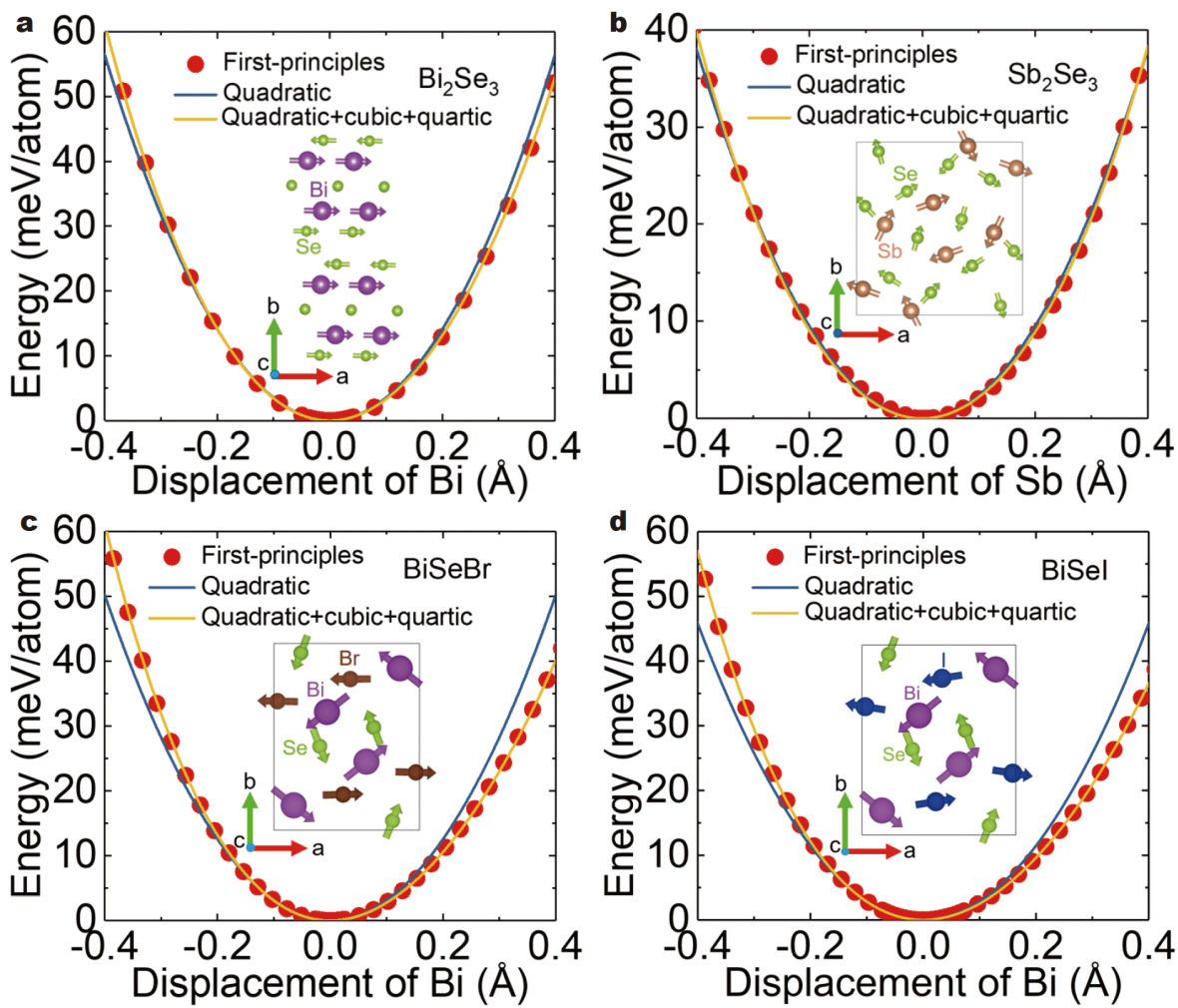

Figure 7 Frozen phonon potentials and displacement patterns of the optical mode calculated by the First-principles. Anharmonic frozen-phonon potentials of (a) $\mathrm{E}_{\mathrm{g}}$ mode in $2 \mathrm{D} \mathrm{Bi}_{2} \mathrm{Se}_{3}$, (b) $\mathrm{A}_{\mathrm{g}}$ mode in $1 \mathrm{D} \mathrm{Sb} \mathrm{Se}_{3}$, (c) $\mathrm{A}_{\mathrm{g}}$ mode in $1 \mathrm{D} \mathrm{BiSeBr}$ and (d) $\mathrm{A}_{\mathrm{g}}$ mode in $1 \mathrm{D}$ BiSeI. The insets are the displacement patterns of the corresponding modes at the $\Gamma$ point. 
dicates larger displacements of $\mathrm{Bi}$ and halogen atoms along the $a$ and $b$ directions (Fig. S7), which was confirmed by temperature-dependent atomic displacement measurements (Figs S8, S9). The temperature-dependent lattice parameters and atomic positions of these four compounds are shown in Fig. S10 and Tables S7-S10. The isotropic displacements of $\mathrm{Bi}$ and $\mathrm{Br} / \mathrm{I}$ in $\mathrm{BiSeBr}$ and $\mathrm{BiSeI}$ increase with temperature and are larger than $\mathrm{Bi}$ in $2 \mathrm{D}$ $\mathrm{Bi}_{2} \mathrm{Se}_{3}, \mathrm{Sb}$ in $1 \mathrm{D} \mathrm{Sb}_{2} \mathrm{Se}_{3}, \mathrm{Cu}$ in $2 \mathrm{D} \mathrm{BiCuSeO}$ [43], and $\mathrm{Pb}$ in $3 \mathrm{D} \mathrm{PbTe} \mathrm{[44]} \mathrm{(Fig.} \mathrm{S9),} \mathrm{indicating} \mathrm{the} \mathrm{soft} \mathrm{chains} \mathrm{in}$ bismuth selenohalides. The larger displacement of the halogen atoms and the weak $\mathrm{Bi}-\mathrm{I} / \mathrm{Br}$ bond may lead to ion migration, as verified by the ionic conductivity of bismuth selenohalides (Fig. S11). Thus, the strong anharmonicity of the optical mode, larger atomic displacement and ion migration all contribute to the extremely low thermal conductivity.

Since the observation of low thermal conductivity in bismuth selenohalides, good thermoelectric transport properties are also expected. The optical absorption spectrum indicates BiSeI is a wide gap semiconductor with a very high electrical resistance (Fig. S12). The thermoelectric properties of the 1D BiSeI-based materials were studied through producing Se vacancies (Figs S13S15 and Table S11). The Se vacancies can effectively boost the electrical conductivity and power factor of BiSeI, especially in the direction perpendicular to the SPS pressure direction. Finally, the maximum $Z T$ of $B \mathrm{BSe}_{0.95} \mathrm{I}$ can reach 0.11 (parallel) and 0.18 (perpendicular) at $573 \mathrm{~K}$ (Fig. S16), attributed to the extremely low thermal conductivity. It can be expected that the thermoelectric performance of the 1D BiSeI-based materials can be improved further through increasing the electrical transport properties in future work.

\section{CONCLUSION}

This work demonstrates that the 1D bulk bismuth selenohalides possess extremely low thermal conductivity $\left(\sim 0.27 \mathrm{~W} \mathrm{~m}^{-1} \mathrm{~K}^{-1}\right.$ at $\left.573 \mathrm{~K}\right)$. By using the first-principles calculations and experimental measurements, the origins of the low thermal conductivity were revealed and compared with (2D) $\mathrm{Bi}_{2} \mathrm{Se}_{3}$ and (1D) $\mathrm{Sb}_{2} \mathrm{Se}_{3}$ compounds. The results indicate that the weak interaction between $1 \mathrm{D}$ chains, lower cut-off acoustic frequency and phonon velocity, strong anharmonicity of both acoustic and optical mode, and larger displacements of $\mathrm{Bi}$ and halogens are responsible for the extremely low thermal conductivity of bismuth selenohalides. The more importance over the material itself is to provide a brand-new strategy to search or design novel materials with intrinsically low thermal conductivity and simple crystal structures. This work opens up prospects of low thermal conductivity materials with potential applications in thermoelectrics and thermal barrier coatings.

Received 13 May 2020; accepted 20 May 2020;

published online 11 June 2020

1 Li S, Zheng Q, Lv Y, et al. High thermal conductivity in cubic boron arsenide crystals. Science, 2018, 361: 579-581

2 Wan C, Qu Z, He Y, et al. Ultralow thermal conductivity in highly anion-defective aluminates. Phys Rev Lett, 2008, 101: 085901

3 Padture NP, Gell M, Jordan EH. Thermal barrier coatings for gasturbine engine applications. Science, 2002, 296: 280-284

4 Zhao LD, Pei YL, Liu Y, et al. $\mathrm{InFeZnO}_{4}$ as promising thermal barrier coatings. J Am Ceramic Soc, 2011, 94: 1664-1666

5 Zhao LD, Lo SH, Zhang Y, et al. Ultralow thermal conductivity and high thermoelectric figure of merit in SnSe crystals. Nature, 2014, 508: 373-377

6 He J, Tritt TM. Advances in thermoelectric materials research: Looking back and moving forward. Science, 2017, 357: eaak9997

7 Tan G, Zhao LD, Kanatzidis MG. Rationally designing highperformance bulk thermoelectric materials. Chem Rev, 2016, 116: 12123-12149

8 Ying $\mathrm{P}, \mathrm{Li} \mathrm{X}$, Wang $\mathrm{Y}$, et al. Hierarchical chemical bonds contributing to the intrinsically low thermal conductivity in $\alpha$ MgAgSb thermoelectric materials. Adv Funct Mater, 2017, 27: 1604145

9 Xiao Y, Zhao LD. Seeking new, highly effective thermoelectrics. Science, 2020, 367: 1196-1197

10 Lindsay L, Broido DA, Reinecke TL. First-principles determination of ultrahigh thermal conductivity of boron arsenide: A competitor for diamond? Phys Rev Lett, 2013, 111: 025901

11 Kang JS, Li M, Wu H, et al. Experimental observation of high thermal conductivity in boron arsenide. Science, 2018, 361: 575578

12 Tian F, Song B, Chen X, et al. Unusual high thermal conductivity in boron arsenide bulk crystals. Science, 2018, 361: 582-585

13 Chen K, Song B, Ravichandran NK, et al. Ultrahigh thermal conductivity in isotope-enriched cubic boron nitride. Science, 2020, 367: 555-559

14 Brown SR, Kauzlarich SM, Gascoin F, et al. $\mathrm{Yb}_{14} \mathrm{MnSb}_{11}$ : New high efficiency thermoelectric material for power generation. Chem Mater, 2006, 18: 1873-1877

15 Zhao J, Hao S, Islam SM, et al. Six quaternary chalcogenides of the pavonite homologous series with ultralow lattice thermal conductivity. Chem Mater, 2019, 31: 3430-3439

16 Miller SA, Gorai P, Ortiz BR, et al. Capturing anharmonicity in a lattice thermal conductivity model for high-throughput predictions. Chem Mater, 2017, 29: 2494-2501

17 Chang C, Zhao LD. Anharmoncity and low thermal conductivity in thermoelectrics. Mater Today Phys, 2018, 4: 50-57

18 Mukhopadhyay S, Parker DS, Sales BC, et al. Two-channel model for ultralow thermal conductivity of crystalline $\mathrm{Tl}_{3} \mathrm{VSe}_{4}$. Science, 2018, 360: 1455-1458

19 Dutta M, Matteppanavar S, Prasad MVD, et al. Ultralow thermal conductivity in chain-like TlSe due to inherent $\mathrm{Tl}^{+}$rattling. J Am Chem Soc, 2019, 141: 20293-20299

20 Samanta M, Pal K, Pal P, et al. Localized vibrations of Bi bilayer 
leading to ultralow lattice thermal conductivity and high thermoelectric performance in weak topological insulator $n$-type BiSe. J Am Chem Soc, 2018, 140: 5866-5872

21 Jana MK, Biswas K. Crystalline solids with intrinsically low lattice thermal conductivity for thermoelectric energy conversion. ACS Energy Lett, 2018, 3: 1315-1324

22 Peng B, Xu K, Zhang H, et al. 1D SbSeI, SbSI, and SbSBr with high stability and novel properties for microelectronic, optoelectronic, and thermoelectric applications. Adv Theor Simul, 2018, 1: 1700005

23 Pele V, Barreteau C, Berardan D, et al. Direct synthesis of BiCuChO-type oxychalcogenides by mechanical alloying. J Solid State Chem, 2013, 203: 187-191

24 Xiao Y, Chang C, Pei Y, et al. Origin of low thermal conductivity in SnSe. Phys Rev B, 2016, 94: 125203

25 He W, Wang D, Dong JF, et al. Remarkable electron and phonon band structures lead to a high thermoelectric performance $Z T>1$ in earth-abundant and eco-friendly SnS crystals. J Mater Chem A, 2018, 6: 10048-10056

26 Chen Y, Wang D, Zhou Y, et al. Enhancing the thermoelectric performance of $\mathrm{Bi}_{2} \mathrm{~S}_{3}$ : A promising earth-abundant thermoelectric material. Front Phys, 2018, 14: 13601

27 Lai W, Wang Y, Morelli DT, et al. From bonding asymmetry to anharmonic rattling in $\mathrm{Cu}_{12} \mathrm{Sb}_{4} \mathrm{~S}_{13}$ tetrahedrites: When lone-pair electrons are not so lonely. Adv Funct Mater, 2015, 25: 3648-3657

28 Wölfing B, Kloc C, Teubner J, et al. High performance thermoelectric $\mathrm{Tl}_{9} \mathrm{BiTe}_{6}$ with an extremely low thermal conductivity. Phys Rev Lett, 2001, 86: 4350-4353

29 Tan G, Hao S, Zhao J, et al. High thermoelectric performance in electron-doped $\mathrm{AgBi}_{3} \mathrm{~S}_{5}$ with ultralow thermal conductivity. J Am Chem Soc, 2017, 139: 6467-6473

30 Kurosaki K, Yamanaka S. Low-thermal-conductivity group 13 chalcogenides as high-efficiency thermoelectric materials. Phys Status Solidi A, 2013, 210: 82-88

$31 \mathrm{He} \mathrm{W}$, Wang $\mathrm{D}, \mathrm{Wu} \mathrm{H}$, et al. High thermoelectric performance in low-cost $\mathrm{SnS}_{0.91} \mathrm{Se}_{0.09}$ crystals. Science, 2019, 365: 1418-1424

32 Qin B, Wang D, He W, et al. Realizing high thermoelectric performance in p-type SnSe through crystal structure modification. J Am Chem Soc, 2019, 141: 1141-1149

33 Savin A, Jepsen O, Flad J, et al. Electron localization in solid-state structures of the elements: The diamond structure. Angew Chem Int Ed Engl, 1992, 31: 187-188

34 Shi X, Chen L, Uher C. Recent advances in high-performance bulk thermoelectric materials. Int Mater Rev, 2016, 61: 379-415

35 Morelli DT, Jovovic V, Heremans JP. Intrinsically minimal thermal conductivity in cubic I-V-VI 2 semiconductors. Phys Rev Lett, 2008, 101: 035901

36 Slack GA. Nonmetallic crystals with high thermal conductivity. J Phys Chem Solids, 1973, 34: 321-335

37 Pei Y, Chang C, Wang Z, et al. Multiple converged conduction bands in $\mathrm{K}_{2} \mathrm{Bi}_{8} \mathrm{Se}_{13}$ : A promising thermoelectric material with extremely low thermal conductivity. J Am Chem Soc, 2016, 138: 16364-16371

38 Ding J, Xu B, Lin Y, et al. Lattice vibration modes of the layered material $\mathrm{BiCuSeO}$ and first principles study of its thermoelectric properties. New J Phys, 2015, 17: 083012

39 Nielsen MD, Ozolins V, Heremans JP. Lone pair electrons minimize lattice thermal conductivity. Energy Environ Sci, 2013, 6: 570-578

40 Barreteau C, Berardan D, Dragoe N. Studies on the thermal sta- bility of BiCuSeO. J Solid State Chem, 2015, 222: 53-59

41 Zhang Y, Ozoliņš V, Morelli D, et al. Prediction of new stable compounds and promising thermoelectrics in the Cu-Sb-Se system. Chem Mater, 2014, 26: 3427-3435

42 Hao S, Shi F, Dravid VP, et al. Computational prediction of high thermoelectric performance in hole doped layered GeSe. Chem Mater, 2016, 28: 3218-3226

43 Vaqueiro P, Al Orabi RAR, Luu SDN, et al. The role of copper in the thermal conductivity of thermoelectric oxychalcogenides: Do lone pairs matter? Phys Chem Chem Phys, 2015, 17: 31735-31740

44 Bozin ES, Malliakas CD, Souvatzis P, et al. Entropically stabilized local dipole formation in lead chalcogenides. Science, 2010, 330: $1660-1663$

Acknowledgements We appreciate the help from Prof. Shubin Yang and Dr. Yongzheng Shi for ionic conductivity measurement. This work was supported by the National Key Research and Development Program of China (2018YFA0702100 and 2018YFB0703600), the National Natural Science Foundation of China (51772012 and 51632005), Shenzhen Peacock Plan team (KQTD2016022619565991), Beijing Natural Science Foundation (JQ18004), China Postdoctoral Science Foundation Grant (2019M650429), 111 Project (B17002) and the National Science Foundation for Distinguished Young Scholars (51925101). Wu H acknowledges the financial support from Singapore Ministry of Education Tier 1 grant (R-284-000-212-114) for Lee Kuan Yew Postdoctoral Fellowship. Wang $G$ is grateful to the High Performance Computing Center of Henan Normal University. Wang D thanks the high performance computing (HPC) resources at Beihang University.

Author contributions Wang D and Zhao LD initiated the work, analyzed the results, and wrote the paper. Wang D and Wang G performed the DFT calculations. Huang Z and Zhao LD synthesized the samples and carried out the thermal and electrical properties measurements. Zhang Y, Wang $\mathrm{H}$ and Pennycook SJ carried out the STEM measurements. He L, Wang H, Deng S, Chen J and He L carried out the high temperature neutron powder-diffraction (NPD) measurements and Rietveld refinements. All authors conceived the experiments, analyzed the results, and coedited the manuscript.

Conflict of interest The authors declare that they have no conflict of interest.

Supplementary information Supporting data are available in the online version.

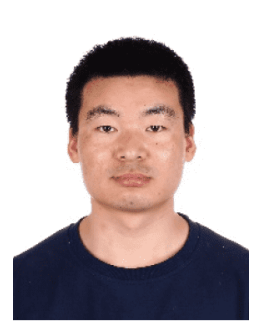

Dongyang Wang obtained his BSc and MSc degrees in physics from Henan Normal University, China in 2014 and 2017, respectively. He obtained his $\mathrm{PhD}$ degree in materials science from Beihang University in 2020. His current research focuses on the exploration and design of low thermal conductivity materials. 


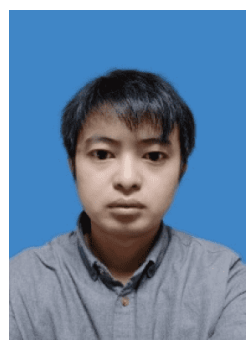

Zhiwei Huang has been a post-doctoral fellow in Prof. Li-Dong Zhao's group at Beihang University since 2018 . He got the BE degree in applied chemistry from Jilin University and $\mathrm{PhD}$ degree in physical chemistry from Dalian Institute of Chemical Physics, Chinese Academy of Sciences in 2018. His research interests are metal chalcogenides-based thermoelectric materials.

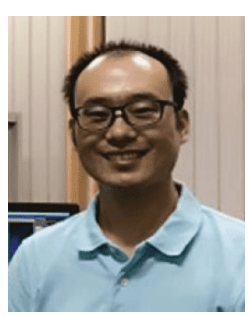

Haijun Wu is a Lee Kuan Yew Postdoctoral fellow at the Department of Materials Science and Engineering, National University of Singapore (NUS), Singapore. He obtained his BSc and MSc degrees from Xi'an Jiaotong University, China in 2009 and 2012, respectively. $\mathrm{He}$ obtained his PhD degree from NUS in 2019. His research interests are STEM and EELS, and structure-property correlation in energy materials, e.g., thermoelectrics, piezoelectrics/ferroelectrics, and functional oxide interfaces.

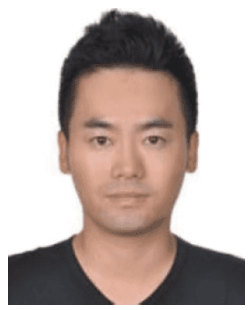

Li-Dong Zhao is a full professor of materials science and engineering at Beihang University, China. He received his $\mathrm{PhD}$ degree from the University of Science and Technology Beijing, China, in 2009. He was a postdoctoral research associate at the Université Paris-Sud and Northwestern University from 2009 to 2014 . His research interests include electrical and thermal transport behaviors in the compounds with layered structures. Group website: http://shi. buaa.edu.cn/zhaolidong/zh_CN/index.htm

\section{具有超低热导率的一维铋硒卤族化合物}

王东洋 ${ }^{1 \dagger}$, 黄志伟 ${ }^{1 \dagger}$, 张杨 ${ }^{3,4}$, 郝丽㷊 ${ }^{5}$, 王广涛 ${ }^{6}$, 邓司浩 ${ }^{7}$, 王洪亮 ${ }^{5}$, 陈洁 ${ }^{9}$, 何伦华 ${ }^{7,8,9}$, 肖宝 ${ }^{10}$, 徐亚东 ${ }^{10}$, Stephen J. Pennycook ${ }^{4}$, 武海军 ${ }^{2,4^{*}}$, 赵立东 ${ }^{1^{*}}$

摘要 本征低热导率材料的研究具有重要的科学意义, 已引起广泛 关注. 本工作报道了一类具有简单一维晶体结构的超低热导率材 料: 铋硒卤族化合物 (BiSeX, X = Br, I). 研究发现, BiSeI的热导率在 $573 \mathrm{~K}$ 仅为 $0.27 \mathrm{~W} \mathrm{~m}^{-1} \mathrm{~K}^{-1}$, 达到了最低本征热导率的理论极限值. 本研究采用第一性原理计算结合粉末中子衍射和变温球差扫描透 射电子显微镜表征, 深入探究了其超低热导率的机制. 研究表明, BiSeX的一维结构赋予了材料低热导特性: 弱的成键特性、低的声 子速度、声学支和光学支的强非简谐性以及 $\mathrm{Bi}$ 和卤族元素较大的 偏移效应, 从而有效阻碍了声子输运, 使得BiSeX具有超低的热导 率. 本研究提出了在具有一维结构的材料中寻找低传导特性的新 思路, 该研究思路在热电材料和热障涂层材料等低热传导需求领 域中具有广阔的应用前景. 